Bristol-Myers Squibb, Boehringer-Ingelheim, Lilly and Novartis, Consultant of: Lilly, Novartis, Janssen, Boehringer-Ingelheim, Amgen, Pfizer and Abbvie, Speakers bureau: Sandoz, Celltrion, Lilly, Novartis, Janssen, Boehringer-Ingelheim, Amgen, Pfizer and Abbvie, Sindhu Johnson Grant/research support from: Boehringer Ingelheim, Corbus Pharmaceuticals, GlaxoSmithKline, Roche, Merck, Bayer, Consultant of: Boehringer Ingelheim, Ikaria, Christian Stock Employee of: Employee of Boehringer Ingelheim, Martina Gahlemann Employee of: Employee of Boehringer Ingelheim, Lizette Moros Employee of: Lizette Moros is an employee of Boehringer Ingelheim, Margarida Alves Employee of: Employee of Boehringer Ingelheim, Maureen Mayes Grant/research support from: Maureen Mayes has received grants from Boehringer Ingelheim, Corbus, CSL Behring, Eicos, and Galapagos, Consultant of: Maureen Mayes has acted as a consultant for Boehringer Ingelheim, Eicos, and Galapagos. She was a member of the SENSCIS trial Steering Committee (Boehringer Ingelheim) DOI: 10.1136/annrheumdis-2020-eular.2907

\section{SAT0346 THE EFFICACY AND SAFETY OF RITUXIMAB IN 27 CASES OF TREATMENT RESISTANT SYSTEMIC SCLEROSIS WITH SEVERE DISEASE ASSESSED BY ACTIVITY SCORES}

Y. Yalçınkaya ${ }^{1}$, B. Artim-Esen ${ }^{1}$, S. Amikishiyev ${ }^{1}$, N. Aliyeva ${ }^{1}$, A. Gul ${ }^{1}$, L. Ocal ${ }^{1}$, M. Inanc ${ }^{1} .{ }^{1}$ Istanbul University, Istanbul Medical Faculty, Department of Internal Medicine, Division of Rheumatology, Istanbul, Turkey

Background: Treatment options for systemic sclerosis (SSc) remain limited especially in severe skin, lung and musculoskeletal involvements. B-cell targeted therapy with anti-CD20 Rituximab (RTX), is widely available, reports from case series are encouraging as a a rescue therapy and might have an improving effect on organ involvement in SSc.

Objectives: We aimed to retrospectively analyze the efficacy and safety of rituximab (RTX) courses in patients with severe systemic sclerosis who were refractory to standard immunosuppressive treatment.

Methods: Twenty-seven SSc patients fulfilling ACR/EULAR classification criteria (2013) who received RTX treatment due to acive disease despite treatment with immunosuppresives were analyzed. Disease activity was evaluated by using EScSG/EUSTAR activity scores prior to and after RTX treatment. Disease severity was also assessed at baseline by Medsger's index.

Results: The demographics and characteristics of SSc patients were as follows: the median age of 50 (30-70), duration of Raynaud's 10 (3-26) and non-Raynaud symptom 8.5 (3-18) years and summarised in table 1 . RTX was given as a single cycle ( 2 infusions of $1000 \mathrm{mg}$ ) in 12 cases, 2 cyles in 5 cases, $\geq 3$ cyles in 10 cases. DMARDs were prescribed in 19 (73\%) patients (14 MMF, 5 MTX) concomitantly with RTX. The main RTX indications were skin and lung involvement $(n=9)$, skin and arthritis $(n=6)$, skin $(n=5)$, lung $(n=3)$, myositis $(n=2)$, cardiac involvement $(n=1)$ and digital vasculopathy $(n=1)$. Medsger severity score was $7.39 \pm 3.091(3-13)$ at baseline.

Table 1. Prevelance of Characteristics of SSc Patients

\begin{tabular}{lc}
\hline & $\mathrm{n}(\%)$ \\
\hline female/male & $25 / 2$ \\
diffuse/limited cutaneous SSc & $22(81.5) / 5(19,2)$ \\
Clinical Characteristics & $12(44,4) / 10(37,1)$ \\
synovitis / flexion contractures & $7(26,9) / 4(15,4)$ \\
tendon friction rubs / myositis & $1(3,8)$ \\
renal crisis & $19(69,2)$ \\
Gl involvement & $16(61,5)$ \\
lung involvement & \\
Serology & $23(85,2)$ \\
ANA & $16(61,5) / 1(3,8)$ \\
Anti-Scl70 / Anti-sentromer & $6(22,2)$ \\
Anti-Ro &
\end{tabular}

CYC / MMF

$19(73,1) / 19(73,1) 12(46,2) / 16(61,5) 27(100)$

AZA / MTX Low dose steroids

Disease activity and severity scores prior to and after RTX were summarised table 2 .

Disease activity scores were improved after RTX in patients who had a median follow-up period of 1 year (0,5-5 years). After RTX treatment, according to EscSG /EUSTAR scores 13 $(\% 46.2)$ and $10(\% 34.6)$ patients out of 26 were assessed as inactive.

Table 2. Disease activity scores prior to and after RTX treatment

\begin{tabular}{llll}
\hline & Prior to RTX $(\mathbf{n}=\mathbf{2 6})$ & After RTX $(\mathbf{n}=\mathbf{1 8})$ & median $\Delta$ Change $(\mathbf{n = 1 8})$ \\
\hline EscSG activity score & $4,89 \pm 1,82(2,0-9,0)$ & $2,37 \pm 1,10(0,50-4,50)$ & $-2,00(P<0,001)$ \\
EUSTAR activity score & $4,57 \pm 2,68(1,0-10,0)$ & $2,30 \pm 2,15(0,0-7,25)$ & $-2,00(P=0,002)$
\end{tabular}

There were severe infecions in 4 patients (Pneumonia in 2, infected digital ulcers in 2) and an episode of sinusitis in one during treatment period. One patient was deceased because of pneumonia and sepsis after the first cycle of RTX.
Conclusion: In our SSc cohort, RTX treatment was used in severe patients, who had predominantly diffuse cutaneous disease with lung and joint involvements, severe vasculopathy and anti-Scl-70 positivity. Concomitant DMARDs were used in three-forth of the patients in addition to RTX cycles. Disease activity scores that assessed retrospectively were shown to be improved after RTX and $37-48 \%$ of the cases were assessed as inactive by using activity scores. Serious infections like pneumonia and infected digital ulcers were observed in $14,8 \%$ of cases during the follow-up. The addition of RTX treatment can be effective in selected patients with active disease despite immunosuppressive therapy.

Disclosure of Interests: None declared

DOI: 10.1136/annrheumdis-2020-eular.6053

\section{SAT0347 $\quad$ AUTOANTIBODIES TO UNCOUPLED RO52 PROTEIN IN PATIENTS WITH ANTI-SYNTHETASE SYNDROME; A POTENTIAL MARKER FOR SUBCLASSIFICATION.}

A. Yoshida ${ }^{1}$, Y. Okazaki ${ }^{1}$, T. Gono ${ }^{1}$, M. Kuwana ${ }^{1} .{ }^{1}$ Nippon Medical School, Department of Allergy and Rheumatology, Tokyo, Japan

Background: Anti-Ro/SS-A (anti-SS-A) antibody is one of myositis-associated antibodies, and is found in patients with anti-synthetase antibodies ${ }^{1}$. Anti-SS-A antibody targets the complex consisting of Ro52 and Ro60 proteins coupled with cytoplasmic non-coding Y-RNAs. Autoantibody against Ro52 uncoupled with Y-RNAs (anti-uncoupled Ro52) is also present in patients with a variety of connective tissue diseases, including myositis ${ }^{2}$. However, the majority of previous studies used enzyme-linked immunoassay (EIA) for detection of anti-Ro52 antibodies, resulting in failure to discriminate between anti-Ro52 antibodies coupled and uncoupled with Y-RNAs. The prevalence and clinical significance of anti-uncoupled Ro52 antibodies still remain unclear in patients with anti-synthetase antibodies.

Objectives: To elucidate clinical relevance of anti-uncoupled Ro52 antibodies in a cohort of anti-synthetase syndrome employing RNA immunoprecipitation assay (RNA-IP) in combination with EIA.

Methods: This is a single-center, cross-sectional study involving 80 patients positive for anti-synthetase antibodies by RNA-IPP. Complete clinical information was obtained from a medical chart review. Serum samples were obtained at first visit and stored at $-20^{\circ} \mathrm{C}$ until use. Anti-SS-A and anti-SS-B antibodies were detected by RNA-IP, and anti-Ro52 and anti-Ro60 antibodies were measured by commercial EIA kits (ORGENTIC, Mainz, Germany). Autoantibodies that immunoprecipitated Y-RNAs regardless of results of anti-anti-Ro52 or anti-Ro60 EIAs were regarded as anti-SS-A antibody, while antibodies that did not immunoprecipitate Y-RNAs but reacted with anti-Ro52 antibodies by EIA were regarded as anti-uncoupled Ro52 antibody. Student's t-test, Mann-Whitney's U test, and Fisher's exact test were employed to compare the clinical features between each group. Cumulative survival rates were compared using log-rank test.

Results: In our cohort of 80 patients with anti-synthetase antibody, mean age at diagnosis was $61 \pm 12$ years, and $76 \%$ were female. Clinical diagnosis was classic dermatomyositis (cDM) in 11, clinically amyopathic dermatomyositis (CADM) in 21, polymyositis (PM) in 11, systemic sclerosis (SSc) in 3, myositis-SSc overlap in 5, interstitial lung disease (ILD) alone in 29, and unclassified in 3. The antigenic specificity of anti-synthetase antibodies included Jo-1 in 19, PL-7 in 12, PL-12 in 9, EJ in 21, OJ in 4, and KS in 16. Anti-SS-A anti-SS-B antibodies were found in $14(17 \%)$ and $2(2.5 \%)$ patients, respectively. The presence of anti-Ro60 and anti-SS-A antibodies was almost concordant $(P<0.0001)$ although the presence of anti-Ro52 and anti-SS-A antibodies was not correlated $(P=0.8)$. This was primarily because of high prevalence $(40 \%)$ of autoantibodies to uncoupled Ro52. Interestingly, prevalence of anti-uncoupled Ro52 antibodies was different among antigenic specificities of anti-synthetase antibodies: high in Jo-1 (58\%) and EJ (55\%), and low in PL-7 (8\%) and OJ (0\%). Gottron's sign/papule was more frequent in patients with anti-uncoupled Ro52 than in those without (61\% versus $28 \%, P=0.005)$, resulting in clinical diagnosis of $\mathrm{CDM}$ or CADM more common in patients with anti-uncoupled Ro52 than in those without $(59 \%$ versus $26 \% ; P=0.003$ ). The prevalence and extent of ILD tended to be greater in anti-uncoupled Ro52-positive versus negative patients, but difference did not reach statistical significance. There were no differences in cumulative survival rates between patients stratified by the presence or absence of anti-uncoupled Ro52 antibodies.

Conclusion: Autoantibodies to uncoupled Ro52 were commonly found in patients with anti-synthetase antibodies. Anti-Ro52 positivity might be useful for subclassifying anti-synthetase syndrome.

References:

[1] McHugh NJ et al. Nat Rev Rheumatol. 2018; 14(5): 290-302.

[2] Schulte-Pelkum J et al. Autoimmun Rev. 2009; 8(7): 632-7.

Disclosure of Interests: Akira Yoshida: None declared, Yuka Okazaki: None declared, Takahisa Gono Speakers bureau: Astellas, and Medical and Biological Laboratories, Masataka Kuwana Grant/research support from: Acetelion, Consultant of: Acetelion, 